Chrisje Brants

Stewart Field

\title{
Les méthodes d'enquête proactive et le contrôle des risques
}

In: Déviance et société. 1997 - Vol. 21 - N4. pp. 401-414.

Citer ce document / Cite this document :

Brants Chrisje, Field Stewart. Les méthodes d'enquête proactive et le contrôle des risques. In: Déviance et société. 1997 - Vol. 21 - N4. pp. 401-414.

doi : $10.3406 /$ ds. 1997.1825

http://www.persee.fr/web/revues/home/prescript/article/ds_0378-7931_1997_num_21_4_1825 


\title{
débat: \\ Les méthodes d'enquête policières proactives et leur contrôle
}

\author{
Mots-Clés: CoOpération policière - Crime - Crime organisé - Droit pénal -

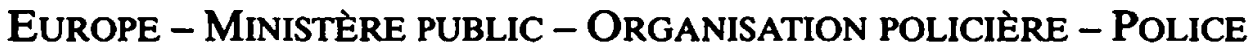

KEY-WORDS: POLICE COOPERATION - CRIME - ORGANISED CRIME - PENAL LAW - EUROPE PROSECUTION - POLICE ORGANISATION - POLICE

\section{LES MÉTHODES D’ENQUÊTE PROACTIVES ET LE CONTRÔLE DES RISQUES}

\author{
C. BRANTS*, S. FIELD**
}

Les méthodes proactives - la collecte secrète de renseignements par la police et/ou par des organismes soit d'Etat, soit privés, afin de faciliter la lutte contre les infractions graves et/ ou les menaces au maintien de l'ordre, au sens large - sont en cours dans tous les pays européens. Elles existent depuis longtemps ${ }^{1}$ mais de récents développements, surtout au niveau de la technologie (électronique) et de la coopération internationale, ont augmenté leur portée et leur étendue, et donc aussi les problèmes que leur usage peut soulever. Les manifestations des méthodes proactives (si l'on peut dire, car vu leur nature secrète, elles se «manifestent» rarement comme telles) changent d'un pays à l'autre selon les différentes constructions sociales du «problème de la délinquance» ou de «la menace contre la société»; elles changent aussi d'un système légal à l'autre. Alors que l'on a beaucoup écrit à ce sujet dans certains pays (notamment aux Pays-Bas, pour des raisons que nous allons développer), dans d'autres, le manque de publications rend l'appréciation de la nature exacte, de l'étendue et de la portée de ces méthodes, très difficile. Ces faits ne permettent guère de faire des comparaisons et ils rendent toute déclaration empirique bien fondée quant à la situation actuelle $^{2}$ quasiment impossible. Cependant cette prise de conscience ne suggère pas que l'on abandonne la recherche de données statistiques ${ }^{3}$; des difficultés pour trouver une définition commune ou des informations précises quant à la quantité de renseignements secrets recueillis dans un pays particulier, ne devraient pas non plus nous dissuader dans notre prise en considération des implications normatives des enquêtes proactives. Il faut aussi étudier les risques potentiels qu'elles posent à un maintien de l'ordre démocratique et au critère d'équité universellement reconnu dans le processus pénal et au-delà. En effet, quelles que

\footnotetext{
- Pompe Instituut, Universiteit Utrecht, Pays-Bas.

** Cardiff Law School, University of Wales, Royaume-Uni.

1 Voir Fijnaut, Marx (1995, 1-16) pour une vue historique d'ensemble.

2 Voir cependant : Fijnaut, Marx, 1995 ; Joubert, 1994 ; pour une comparaison des contrôles proactifs et de leurs effets sur la procédure pénale dans des pays avec des systèmes légaux différents (l'Angleterre et le Pays de Galles, les Pays-Bas) : Brants, Field, 1995 ; pour une comparaison des pouvoirs policiers dans plusieurs pays européens : Bevers, Joubert, 1994.
} 
soient les particularités d'un système pénal, légal ou d'ordre social au niveau national, certaines activités (telles que: viser secrètement des individus et des groupes considérés potentiellement dangereux pour la société; recueillir et conserver des renseignements secrets sur ceux-ci et les utiliser pour rendre les cibles inoffensives) s'accordent mal avec les notions fondamentales des règles de droit - en termes continentaux, Rechtsstaat - et des droits de l'Homme.

Dans cet essai, nous nous proposons d'examiner les méthodes policières proactives modernes et leurs implications, en définissant d'abord les manières différentes dont le terme est utilisé, et en donnant les grandes lignes des problèmes potentiels qui se rattachent aux différents aspects de la question. Un bref condensé des événements aux Pays-Bas montre que des questions sur les méthodes policières proactives en rapport avec le crime organisé, ont provoqué un furieux débat politique et public. Dans ce contexte, nous discuterons plus largement ce que l'usage des méthodes proactives peut représenter pour la surveillance et le contrôle de la police et pour les droits fondamentaux (procès équitable) formulés dans l'article 6 de la Convention Européenne des droits de l'Homme et des libertés fondamentales. Si le débat aux Pays-Bas reflète un problème typiquement hollandais, les problèmes sous-jacents sont présents dans tout pays où la légitimité du système de justice pénale dépend de sa capacité à maintenir l'ordre et la légalité et ceci, selon les règles de droit.

Cependant, si les problèmes des méthodes policières proactives sont clairement illustrés dans leur implications pour le processus pénal, ils ne se limitent en aucun cas aux questions nationales de procédure pénale. D'une part, ils transcendent le «national», comme on le remarquera en examinant brièvement les accords de Schengen et le traité de Maastricht avec son « $3^{e}$ pilier» sur le contrôle supranational du crime. D'autre part, ils transcendent les questions de droit pénal. Après un examen rigoureux, l'on voit, en effet, que de par leur nature, les méthodes proactives en tant qu'instruments de contrôle, ne sont pas nécessairement liées au contrôle de la délinquance (même si, pour des raisons de légitimité politique, leur objet est souvent défini en termes de délinquance). Ces méthodes fournissent un moyen de surveiller le comportement d'individus ou de groupes qui, de fait, ont menacé l'ordre social établi ou qui menacent de le faire ; mais la surveillance concerne aussi ceux qui sont considérés comme potentiellement dangereux (que ce soit à cause de leur nationalité, ethnie, liens familiaux ou sociaux, conduite antérieure, convictions politiques, etc.). L'enjeu principal est donc l'avenir du maintien de l'ordre dans la société (européenne) en général, et la question de savoir comment, et même si, nous, citoyens de cette société, serons capables de contrôler la police et si nous sommes prêts à payer le prix pour obtenir paix et sécurité dans nos pays et dans «l'Europe-forteresse».

\section{Le concept des méthodes policières proactives}

Les méthodes policières proactives se définissent de plusieurs façons et les différentes définitions du concept dépendent du contexte dans lequel elles sont appliquées, bien qu'elles soient toutes apparentées. Tout d'abord, il s'agit de l'usage de certaines méthodes et techniques dans le contexte d'une enquête criminelle: l'observation, la surveillance, l'infiltration et autres opérations sous couverture, l'interception de communications (écoutes téléphoniques, appareils d'écoute). Nombre de ces méthodes requièrent des appareils techniques et électroniques d'une grande complexité. Elles ont ceci en commun: elles ne sont efficaces que si elles restent secrètes. Etant secrètes de nature, ces méthodes transgressent le droit fondamental de protection contre l'intrusion par l'Etat dans la vie privée (droit garanti par l'article 8 de la CEDH) sauf lorsqu'elles se basent sur la loi et sont néces- 
saires à la protection de la démocratie ${ }^{4}$. Il est certainement possible de donner une base légale à ces méthodes (et même les garanties correspondantes contre leur abus, requises par la Cour Européenne), et beaucoup de pays européens, si pas tous, l'ont fait jusqu'à un certain point. D'ailleurs, bien que la nécessité du secret pose de sérieux problèmes aux droits de la défense, les méthodes proactives, lorsqu'elles sont légalement approuvées, servent à recueillir des preuves contre les personnes soupçonnées d'avoir commis un crime ; dans certains cas, l'on peut même considérer que leur usage, dans ce type d'enquêtes criminelles, est justifié, vu les besoins d'une société démocratique.

Cependant, dans sa deuxième définition, proactif signifie le contraire de réactif : la collecte de renseignements sur des individus et des groupes avant qu'un délit n'ait été commis. A présent, il est bon de rappeler que le terme «mainticn de l'ordre» se réfère aux moyens dont dispose un Etat pour parer aux dangers provenant de l'intérieur de ses frontières. Comme seul l'Etat a le droit à l'usage ou à la menace de l'usage de la force, «maintien de l'ordre » est le plus souvent associé au droit pénal ou au maintien direct de l'ordre alors qu'il ne s'y limite nullement. Dans le second sens, les «méthodes policières proactives» touchent visiblement les limites du processus pénal et vont même au-delà de l'action directe. Dans beaucoup de cas, la loi donne aux autorités dans le système de droit pénal et en dehors du système (police de la circulation, autorités des frontières, douaniers, inspecteurs du fisc), le pouvoir d'obliger un citoyen ou un organisme (par ex. une banque) à fournir des renseignements sur eux-mêmes ou sur d'autres et elle permet à ces autorités d'enquêter sur des comportements, sans rapport avec un délit ni en vue d'une enquête criminelle. De telles activités ne sont pas nécessairement secrètes mais elles peuvent l'être. De plus, bien que des renseignements recueillis par les organismes d'Etat à des fins visiblement autres que celles d'une enquête criminelle soient traditionnellement considérées comme séparées du droit pénal et gouvernées par d'autres lois, il arrive souvent que ces renseignements soient transmis aux autorités de la justice pénale, permettant ainsi de contourner les garanties plus strictes de la procédure pénale $e^{5}$. Cela vaut aussi pour un type d'enquête secrète pas toujours reconnue en tant que méthode policière proactive parce que souvent liée à l'espionnage et au contre-espionnage et étant dans les mains d'organismes de renseignements (militaires) distincts - la surveillance et le contrôle d'activités terroristes dans lesquels la ligne entre enquête criminelle et contre-terrorisme peut être très trouble. Le Security Services Act, en Grande-Bretagne, est un exemple classique du manque de clarté de ces distinctions : le MI 5 (les services de sécurité interne) peut, en effet, utiliser ses pouvoirs de surveillance (pouvoirs extensifs dont ils n'ont que très rarement à rendre compte de l'utilisation) pour confronter non seulement le terrorisme mais aussi tout type de «crime grave» (au sens le plus large) ${ }^{6}$.

Si les méthodes policières proactives sont utilisées dans le contexte du droit pénal proprement dit et en tant que moyen de recueillir des renseignements sur des délits criminels qui n'ont pas encore été commis, alors nous devons mettre en question leur base légale et leur objet. Alors que la prévention du crime dans ce sens pourrait aussi justifier l'usage de méthodes incursives, le concept de Rechtsstaat en Europe continentale exige, dans les cas d'usage de ces pouvoirs dans les enquêtes criminelles, un soupçon raisonnable qu'un délit a été commis ou, tout au moins, va être commis. Ceci représente un seuil qui active simulta-

4 Article 8 paragraphe 2 CEDH. Voir aussi Kruslin vs France, CEDH 24 avril 1990, Série A, Nos 176-A et 176-B.

5 La zone la plus problématique ici est le droit de garder le silence et de n'être pas tenu à fournir des preuves contre soi-même. Cf. Funke vs France 25 décembre 1993, série A, № 256A.

6 Cf. Greer, 1995. 
nément les pouvoirs policiers et les droits des prévenus. Les pays de droit coutumier ont un concept résiduel de la liberté, accompagné d'une attitude sceptique quant à l'efficacité des abstractions «arrêtées» des droits constitutionnels - quand appliqués à l'Etat - ; cette attitude dicte que la police peut faire tout ce qui n'est pas spécifiquement illicite. Au contraire, la base même du Rechtsstaat continental (et, ajoutons, de la Convention Européenne) dicte que toute infraction aux libertés et aux droits fondamentaux doit se baser sur la loi. Si, dans les pays de droit coutumier, tels que la Grande-Bretagne, les méthodes policières proactives existent dans un vide juridique, dans les pays continentaux, elles imposent un important problème de légalité avec une dimension supplémentaire lorsqu'elles sont utilisées dans des enquêtes criminelles avant l'établissement requis d'un soupçon raisonnable ${ }^{7}$.

Quant à l'objet de la collecte proactive de renseignements, il peut être ou ne pas être de futures poursuites pour délit criminel, sur base de renseignements ainsi obtenus. Hoogenboom suggère que la collecte de renseignements sans poursuites judiciaires ultérieures, est une des caractéristiques de la définition des méthodes proactives ${ }^{8}$. Cependant, l'aboutissement à des poursuites à la suite de l'obtention de renseignements dépend de la situation : aux Pays-Bas, par ex., certaines collectes proactives de renseignements sont utilisées pour des poursuites mais, selon le cas, beaucoup d'autres ne le sont pas. En France, la pénétration de la police dans des sectes religieuses a parfois été utilisée comme moyen de recueillir des renseignements pour déterminer si ces sectes avaient droit au rang de "religion respectable» : par ex., le droit de se réunir dans des édifices publics. La sphère d'activités des Renseignements Généraux est aussi étendue mais indirectement reliée aux poursuites judiciaires ${ }^{9}$. Il faut aussi tenir compte de la politique pénale : au Royaume-Uni, les méthodes proactives sont toujours considérées (du moins par la police régulière) comme moyens de recueillir des preuves préliminaires, en vue de poursuites judiciaires. Donc, l'idée d'un objet autre que des poursuites ne devrait pas faire partie intégrale de la définition des méthodes proactives, mais devrait plutôt être considérée comme un développement apparenté qui souligne le potentiel de ce type de méthode policière et aggrave considérablement les problèmes de contrôle.

Au cas où les poursuites sont le but visé, la légalité de la méthode d'enquête et la fiabilité des renseignements obtenus (c'est-à-dire les preuves) joueront un rôle dans le procès et seront l'objet - du moins en théorie - d'un examen rigoureux par les autorités hiérarchiquement supérieures à la police, et/ou par la défense et le tribunal. Mais si les informations ainsi recueillies sont destinées à être utilisées comme un moyen de perturber les activités de certains individus et de certains réseaux (criminels) sans jamais les amener devant un tribunal ou même préparer une action à leur encontre, si elles sont stockées en vue d'une possible utilisation future, ou sont simplement destinées à surveiller le comportement et les mouvements de ces cibles, la question qui se pose est alors de savoir comment et par qui ces activités policières doivent elles-mêmes être surveillées et contrôlées. Les garanties de la procédure pénale sont telles qu'elles ne fonctionnent qu'au sein du processus pénal, et n'y restent que lorsqu'elles sont respectées. Sans elles, comment peut-on savoir pourquoi et comment les renseignements sont recueillis, par qui, dans quel but, quelles sont les cibles, qui les a sélectionnées et pour quelles raisons ? C'est l'inquiétude du public à propos de ce type de questions qui a provoqué une enquête parlementaire aux Pays-Bas sur la légalité des pratiques policières.

$7 \quad$ Brants, Field, 1995, 46. Des agents américains du Drug Enforcement Administration, travaillant en Europe occidentale, sur le continent, se sont souvent plaint de la préoccupation européenne à propos de la légalité, qu'ils considèrent comme un obstacle incompréhensible à la réussite du maintien de l'ordre avec des moyens proactifs, à leurs yeux, tout à fait justifiables ( $c f$. Nadelman, 1993, chap. 4).

Hoogenboom, 1995, 570.

9 Monjardet, Lévy, 1995, 34-36. 


\section{Les méthodes policières proactives dans la procédure pénale et le cas des Pays-Bas}

Aux Pays-Bas, comme dans d'autres pays d'Europe occidentale, l'usage de méthodes d'enquête proactives (agents sous couverture, indicateurs, surveillance électronique) s'est développé à partir des années 1970, à la suite de et parallèlement aux activités redoublées du DEA américain en Europe dans la guerre contre la drogue. En effet, le DEA n'a pas seulement eu des contacts réguliers avec les forces de l'ordre européennes mais a cherché à influencer leurs méthodes en fournissant un modèle américanisé du maintien de l'ordre et de son idéologie qui ne tenaient guère compte des exigences légales européennes ${ }^{10}$. Peu de méthodes proactives utilisées par la police hollandaise avaient une base légale ${ }^{11}$, même si elles étaient de plus en plus réglées par la sanction des procureurs et par la Cour Suprême hollandaise. En même temps, l'emphase sur l'objet des méthodes proactives a changé au fil des années ; au départ, ces méthodes étaient utilisées pour faciliter l'arrêt et les poursuites de personnes soupçonnées d'être mêlées à un crime lié à la drogue ; ensuite, il fut question de coordonner la collecte de renseignements sur le crime organisé en général - d'abord pour aller plus loin que les individus et ainsi accéder aux personnes contrôlant l'organisation, puis pour perturber des réseaux entiers, et enfin, pour accumuler systématiquement des données sur des groupes potentiellement liés à des activités criminelles (futures).

Ni la portée de ces opérations, ni le fait que l'usage de ces méthodes d'enquête (devant rester secrètes, même au cours du procès) ébranlait fondamentalement les garanties traditionnelles de la procédure pénale, ne furent vraiment reconnus. Cependant, en 1993, à Amsterdam, le chef de la police, le maire et le procureur général ont annoncé publiquement le démantèlement d'une équipe d'enquête régionale, à la suite d'usage de méthodes dont ils ne voulaient plus être tenus responsables. Cette déclaration a déclenché un scandale qui a abouti à une enquête parlementaire au sujet de la nature, de la portée et de la légalité des méthodes policières d'enquête. La commission d'enquête (Commission Van Traa) a tenu des séances publiques télédiffusées, a délégué beaucoup de recherches, a entendu des centaines de témoins dans un rapport composé de onze lourds volumes ${ }^{12}$. La commission a trouvé des preuves d'un usage à grande échelle de méthodes d'enquête déclarées (dans un rapport gouvernemental ultérieur) «inacceptables en termes de Rechtsstaat et en termes de légalité " ${ }^{13}$. Elle a aussi remarqué une détérioration fondamentale dans les formes traditionnelles du contrôle hiérarchique de la police par le procureur général.

En réaction à un courant de panique morale face à l'étendue du crime organisé aux Pays-Bas («une réaction démesurée » d'après le rapport mentionné plus haut) ${ }^{14}$, la police avait eu recours à, entre autres : la surveillance électronique, des appareils d'écoute et la mise sous écoute sans mandat; des effractions, à la recherche de preuves qui seraient «découvertes » ultérieurement, lors de perquisitions légales, des livraisons contrôlées qui amenaient de grandes quantités de drogue à être écoulées dans le pays, en vente libre (et, lorsque le «contrôle» passait de la police aux agents infiltrés, la marchandise était souvent "perdue »), des marchés avec des indicateurs criminels qui leur permettaient de conserver

10 Voir Nadelman, 1993, chap. 4.

11 Avec l'exception notable des écoutes téléphoniques.

12 Inzake Opsporing, 1996.

13 Ministry of Justice, 1996, 3.

14 Pour un résumé de la construction sociale du problème du crime organisé et la part prédominante jouée par la police elle-même en fournissant des «preuves empiriques» pour soutenir le point de vue que le pays tombait rapidement dans les mains d'une organisation «pieuvre», voir Beetstra et al., 1994. 
les recettes de crimes commis, avec la connaissance et l'approbation de la police, la diffusion de fausses informations à propos des enquêtes proactives par le biais de journalistes crédules, et le financement secret de nouvelles opérations policières proactives et sous couverture à partir de fonds obtenus grâce à des transactions liées au trafic de drogue, et manigancées par la police et ses indicateurs. Ces opérations de surveillance pouvaient durer plusieurs mois. Il y avait un soupçon raisonnable que certaines personnes ciblées avaient commis de graves délits ; dans certains cas, le soupçon était né après les surveillances ; et enfin, parfois il semblerait que l'on ait choisi la cible pour ses présumés rapports, même minimes, avec le crime organisé. L'objet de ce type de collecte de renseignements n'était qu'occasionnellement les poursuites judiciaires à l'encontre de la cible.

Ce changement d'objectif correspond de près au déclin du contrôle interne des enquêtes policières par le Ministère Public. Dans un système modérément inquisitorial tel que celui des Pays-Bas, les garanties mises en place pour assurer la recherche de la vérité et l'équité du processus pénal se composent d'un mélange de contrôle hiérarchique entre, et au sein de la police et les services du Ministère Public, de la participation de la défense et de l'élaboration d'un dossier complet et véridique sur lequel la cour pourra baser son examen des preuves ${ }^{15}$. Au début d'un procès, le rôle du procureur est de diriger l'enquête policière en fournissant les grandes lignes d'action opportune en général ainsi qu'en commandant ou en approuvant des enquêtes dans des cas particuliers. La participation de la défense est encore très limitée à ce stade (par exemple, elle n'a pas le droit d'accès au dossier de la police contenant l'élaboration des preuves). L'accusation décide, à partir de l'enquête policière rapportée dans le dossier judiciaire, s'il faut mener l'affaire plus loin ; c'est seulement après l'inculpation officielle du prévenu que le dossier de l'accusation contenant le casier judiciaire devient accessible à la défense. S'il y a lieu de prendre des mesures importunes qui transgressent les droits fondamentaux, un juge d'instruction est censé les approuver au préalable ; une enquête judiciaire préliminaire est alors ouverte. A ce stade, les droits de participation de la défense s'accroissent, bien qu'ils puissent être réduits «dans l'intérêt de l'enquête». Néanmoins, l'accès au dossier, le droit de demander l'audition d'un témoin et de l'interroger, le droit de demander de nouvelles enquêtes (sans avoir le droit de les faire ouvrir), tous ces éléments renforcent le rôle de la défense d'assurer que ni l'enquête ni les preuves ne contiennent des lacunes ou des irrégularités et que le dossier en donne un compte-rendu complet et correct.

Durant le procès, les principes d'immédiateté et du contradictoire ${ }^{16}$ sont en rapport avec le type de droits de la défense que l'on associe habituellement au système du débat contradictoire : non seulement y a-t-il le droit (typiquement inquisitorial) de demander à la cour de renvoyer le procès au juge d'enquête et, mais la défense peut aussi demander l'audition et l'interrogation des témoins, soulever des questions sur la légalité de l'obtention des preuves et/ou sur la méconduite de la police - le tout basé sur un dossier auquel la défense a eu préalablement et pleinement accès. Des lacunes dans le dossier, des inconsistances, des indications de délits policiers ou de négligence flagrante des droits de la défense pourraient conduire au rejet de l'affaire si la cour décide d'exclure les preuves obtenues illicitement ou encore, de rejeter le procès de l'accusation au nom de nullité de procédure et de négligence flagrante du cours normal de la procédure pénale.

Alors qu'en théorie, il incombe - mais il serait également bien avisé de le faire - à un procureur de s'informer des développements au cours de l'enquête préliminaire, son aptitude à le faire réellement dépend des informations que la police veut bien lui donner.

15 Pour le rôle essentiel de la surveillance hiérarchique et le dossier en procédure inquisitoriale, voir Damaska, 1986, chap. 1 et 11.

16

NDLT : en français dans le texte. 
L'usage de méthodes secrètes et illicites n'est pas le genre de renseignement que la police aime donner. Plus particulièrement, parce que la police cherche non seulement à cacher ses activités illicites mais aussi à ce que cette illégalité ne puisse nuire à l'accusation, parce qu'elle cherche à protéger ses sources de renseignements (indicateurs, agents sous couverture), elle ne peut risquer une entière divulgation à la défense.

Les procureurs n'étaient, en fait, pas informés des opérations policières sauf quand leur coopération ou, à travers leurs offices, celle d'un juge d'enquête, était requise pour obtenir des preuves légalement - ou pour blanchir des preuves obtenues illégalement dans un cas bien particulier. Avec l'évolution des méthodes policières proactives (la collecte de plus en plus importante de renseignements non reliés à une affaire spécifique), la nécessité d'informer l'accusation n'a cessé de diminuer. En effet, la nature de plus en plus secrète de telles opérations a engendré une politique de «nécessité d'informer» même dans les secteurs spécialisés de la police - et pour l'extérieur, qui faut-il informer si un procès n'a pas lieu ? Il semble y avoir un mécanisme inhérent aux méthodes proactives : plus elles s'éloignent des limites de la légalité, plus elles échappent au contrôle hiérarchique. Moins il y a de personnes informées, plus le terrain d'activités policières autonomes s'élargit en même temps que le risque de corruption d'agents individuels augmente ${ }^{17}$.

Même lorsque les affaires sont traduites devant le tribunal, les méthodes proactives ont des conséquences néfastes sur les garanties du cours de la justice : le droit du prévenu le plus poussé au débat contradictoire de prendre connaissance des preuves contre lui et de les examiner, droit garanti par la notion de procès équitable contenu dans l'article 6 de la CEDH, et le rôle actif du tribunal dans sa recherche de la vérité et du bon déroulement du cours de la justice. La Commission Van Traa n'a pas examiné à fond ces conséquences mais elles sont plus que claires dans la jurisprudence de la Cour Suprême hollandaise concernant plusieurs affaires célèbres. En ce qui concerne la défense, il y a deux problèmes. D'abord, la question du timing : au moment où les droits de la défense interviennent, beaucoup d'événements clés auront déjà eu lieu, qui auront déterminé de façon substantielle la conduite et souvent, l'aboutissement du procès. Dans ce cas, un tribunal hollandais peut poursuivre avec la présomption que le dossier est complet et exact, que les preuves ont été obtenues légalement et que les témoins sont fiables, à moins que la défense montre des doutes substantiels prima facie. Evidemment, pour y arriver, celle-ci doit avoir accès à tous les aspects de l'affaire, y compris les méthodes utilisées pour obtenir les preuves, la faculté d'entendre des témoins, de les examiner et de connaître leur identité. Ces droits de la défense vont à l'encontre de la nécessité du secret des méthodes proactives ${ }^{18}$, et malgré les conditions de l'article 6 de la CEDH, avec le temps, ils se sont érodés dans la jurisprudence. De leur côté, les tribunaux ont été plus que prêts à accepter les rapports de la police au pied de la lettre, questionnant rarement les preuves cruciales de l'accusation ${ }^{19}$. Il faut ajouter la générosité de la Cour Suprême dans sa large accepta-

17 En effet, plusieurs cas d'implication avec le crime organisé ont été découverts.

18 Il est indicatif que, dans le système de débat contradictoire du Royaume-Uni, l'exigence de divulgation à la défense eut pour résultat de forcer la police à abandonner certaines affaires et que, suite à des plaintes arguant du fait que le contrôle du crime devenait alors impossible, les règles ont été modifiées (le Criminal Procedure and Investigations Act 1996 a reçu le consentement royal en juillet 1996) et le droit automatique de la défense à la divulgation totale n'existe plus. Voir Brants, Field, 1995, 66-67.

19 Bien que, pour être juste, lorsqu'en face de revendications de la police et de l'accusation que de futures opérations ou même la vie de policiers ou de civils seraient en danger si certains faits étaient révélés, les juges ont souvent été obligés de décider que même un examen superficiel de la légalité et de la fiabilité des preuves était trop risqué. Pour une vue d'ensemble de la jurisprudence en Hollande qui a progressivement miné les droits de la défense (et des tribunaux) à la divulgation, voir Brants, Field, 1995, 58-61. 
tion des méthodes proactives extralégales et celle des tribunaux vis-à-vis des rapports de police commençant par ces lignes : les renseignements du CID montrent que... sans jamais demander pourquoi, comment et par qui ces renseignements avaient été obtenus ; ces attitudes ont certainement encouragé les forces de l'ordre à penser qu'il n'y avait rien de fondamentalement mauvais à garder ce genre d'information pour eux.

La Cour Suprême a exigé un rôle plus actif de la part des juges d'instruction dans l'examen du réquisitoire de l'accusation après avoir été mise devant l'évidence que la police, dans plusieurs affaires - parfois à la connaissance de l'accusation, le plus souvent de sa propre initiative - avait délibérément omis des faits essentiels du dossier, falsifié des dossiers, et parfois, maquillé des preuves. Bien que ces directives aient signifié une certaine restauration du rôle traditionnel de surveillance des juges d'instruction en Hollande dans la procédure pénale, c'est beaucoup moins vrai pour les droits de la défense. Dans les affaires où la collecte proactive de renseignements est en jeu, le système se borne à respecter le strict minimum des exigences de la Cour Européenne - et encore - c'est-à-dire que la défense doit pouvoir contester les preuves à un certain moment de la procédure ${ }^{20}$, mais ni en public, ni durant le procès alors qu'une divulgation, même limitée, dépend souvent d'une requête de la défense. C'est donc au défendeur qu'il incombe de démontrer la pertinence du matériel pour son procès, avant d'y avoir accès.

Entre-temps, le parlement hollandais a accepté la plupart des recommandations de la Commission Van Traa et prépare une législation sur les méthodes proactives qui attentent aux droits fondamentaux. Il y a des projets d'introduire une surveillance plus stricte des preuves que la police et l'accusation peuvent vouloir garder secrètes (bien que ces projets n'incluent pas un examen par la défense) et il est question de réorganiser les forces de l'ordre et le Ministère Public. Il faut encore voir si cet effort sera plus que cosmétique tandis que la législation risque de devenir un moyen de légitimer des méthodes d'enquête importunes et qui vont loin, tout en ayant qu'une efficacité largement symbolique. Bien sûr, dans un certain sens, les Pays-Bas ont eu cette chance que la dynamique du scandale ait au moins porté sur la place publique le problème des méthodes policières proactives au service de la justice criminelle. Mais le revers de la pièce existe aussi. Les résultats de la Commission Van Traa démontrent combien la fin peut en venir à justifier les moyens, ce qui altère fondamentalement l'équilibre précaire du processus pénal et des relations entre ses participants. Plus important, les fondations mêmes du système - la croyance dans sa faculté d'auto-contrôle - ont été sérieusement secouées tandis que le spectacle public d'une police faisant sa propre loi a jeté un doute sur la légitimité du contrôle du crime : dans l'idéologie du Rechtsstaat, une police convenablement réglementée est censée soutenir la démocratie, et non la menacer potentiellement. Il reste à voir si la confiance publique dans l'intégrité du système pénal en général - traditionnellement une caractéristique de la société hollandaise - a été durablement minée. Dans d'autres pays, de telles périodes d'efforts de réglementation des forces de l'ordre n'ont guère duré. En 1991, en Angleterre et au Pays de Galles, une réaction à une série d'erreurs judiciaires a amené, à la fois la création d'une commission extraparlementaire et la production d'importants changements en jurisprudence qui ont renforcé les droits de la défense de connaître ce qui eut lieu lors du déroulement de l'enquête criminelle. Mais dès 1993, des inquiétudes au sujet de la délinquance juvénile - attisées par l'affaire Jamie Bulger - ont créé des pressions politiques qui ont causé, en 1996, l'érosion de ces droits au profit des méthodes policières proactives. Il semble que la politique change avec chaque nouveau scandale.

$20 \quad$ Voir entre autres Kostovski vs Pays-Bas CEDH 20 novembre 1989, Série A, No 106. 


\section{Au-delà de la procédure pénale}

Etant donné les circonstances dans lesquelles le scandale a éclaté, l'on peut comprendre que la Commission Van Traa se soit préoccupée presque exclusivement du rôle de la police et des méthodes proactives dans le contexte des enquêtes criminelles sur le crime organisé aux Pays-Bas. Certaines questions ayant un rapport avec ce problème n'ont donc pas été abordées. L'une d'elles est le choix proactif des cibles à surveiller qui engendre une dynamique singulière d'exclusion et de contrôle d'individus et de groupes en marge de la société. Une autre est que, malgré un regard optimiste sur la prise de conscience européenne qui place les droits et libertés de la Convention Européenne des droits de l'Homme au centre d'un mouvement convergent dans la procédure pénale ${ }^{21}$, l'européanisation a cependant un côté négatif : la convergence des méthodes policières proactives dans un contexte européen au niveau international, institutionnalise la collecte et l'échange de renseignements dont une partie seulement se rapporte à des activités criminelles. De plus, ce mouvement qui dépasse les limites nationales vient aggraver le problème de responsabilité. En effet, les forces de l'ordre mais aussi les organismes fixant les responsabilités de la police sont traditionnellement organisés autour d'organes d'Etatnation. Dépasser les limites nationales, c'est aussi échapper aux organismes de contrôle.

\section{Le contrôle des risques}

Dès 1985, commentant les développements en Allemagne, Riehle entrevoyait un nouveau type de maintien de l'ordre qui naîtrait à partir de l'utilisation d'agents sous couverture dans les cas où leur choix d'une cible ne dépendrait plus du critère de soupçon légitime justifiant une enquête criminelle ${ }^{2}$. Bien que ses préoccupations étaient principalement liées aux problèmes de provocation (réussie) par la police, d'individus ciblés par des agents sous couverture pour la simple raison qu'ils étaient vus comme des criminels potentiels, Riehle allait plus loin dans son raisonnement dans le sens où il prédît l'émergence progressive d'une "politique du contrôle des risques» : l'usage par l'Etat de ses pouvoirs exclusifs de contrôle afin de maîtriser certains secteurs de la société. Les individus désignés comme posant un risque au maintien de l'ordre, seraient probablement tirés des marges de la société et reconnaissables à la couleur de leur peau, à leurs affiliations politiques, à leurs condamnations antérieures, aux contacts personnels qu'ils entretiennent avec des suspects, etc. Toujours selon Riehle, ce type de politique entraînerait la marginalisation et jusqu'à la criminalisation, non d'un comportement mais bien d'individus membres de certains groupes au sein de la société et éventuellement de ces groupes eux-mêmes. Finalement, le facteur déterminant dans cette sélection des cibles, ne serait plus leur présumée prédilection à commettre un délit mais uniquement leur identité.

Une dizaine d'années plus tard, maintenant que les méthodes d'enquête proactives vont bien au-delà de l'utilisation d'agents sous couverture, qu'elles sont largement répandues, internationalisées, qu'elles s'appuient de plus en plus sur l'informatisation des données, le «contrôle des risques» est devenu un fait accompli. Cependant, la reconnaissance non-déguisée de cette politique au niveau national est obscurcie par des discussions de problèmes posés par les "groupes à risque en termes de contrôle du crime ; au niveau international, c'est le débat sur la coopération des forces de l'ordre contre le crime orga-

\footnotetext{
21 Voir entre autres Delmas-Marty, 1995.

22 Riehle, 1985.
} 
nisé qui domine, en tant que conséquence naturelle et désirable d'une Europe unifiée. Un détail du compte-rendu de la Commission Van Traa sur la situation aux Pays-Bas illustre comment le choix proactif de cibles - dans le cas présent, une partie de la population turque émigrée aux Pays-Bas - peut produire sa propre légitimation. Le rôle actif de groupes criminels organisés turcs dans le trafic de la drogue est bien connu à Amsterdam. Les criminologues chargés par la Commission Van Traa d'enquêter sur le problème du crime organisé à Amsterdam, ont étudié les données policières pour découvrir l'étendue des opérations dans cette ville, celles-ci suggéraient qu'environ 7000 Turcs avaient des connexions avec le trafic de la drogue. Cependant, après un examen plus rigoureux, il n'y avait d'indications substantielles de connexions (et donc pas nécessairement d'implication de nature criminelle) que pour $25 \%$ des individus en cause. D'autres avaient été inclus parce qu'en tant que Turcs, ils fréquentaient certains cafés et autres endroits «suspects», avaient des contacts avec des «suspects», ou faisaient partie de leur famille, etc. De plus, les données laissaient apparaître un nombre important de «doublons». De même, les noms «sans tache» n'étaient pas régulièrement effacés de la banque des données. Une fois sur la liste, une personne quelconque était devenue une cible légitime de surveillance, tandis que les données elles-mêmes servaient de justification à la politique de surveillance de ce segment de la population immigrée ${ }^{23}$.

\section{Les dimensions de l'internationalisation}

La tendance fondamentale des méthodes policières proactives à dépasser les limites réglementaires traditionnelles de la procédure pénale et à engendrer une dynamique du contrôle des risques, prend une ampleur supplémentaire dans ses développements internationaux ${ }^{24}$. Le contrôle de l'Europe est décidément un vrai patchwork, dépendant d'un réseau complexe d'organismes (officiels ou non) au sein desquels la coopération des forces de l'ordre semble se développer de la même manière ${ }^{25}$, mais rapidement et dans une direction décidément perturbante. Les détails complexes de ce patchwork dépassent de loin l'objet de cet article; il nous suffira ici de dire que les structures sans aucun doute les plus importantes au sein du contexte de l'Union Européenne trouvent leur source dans le traité de Schengen, le «troisième pilier» du traité de Maastricht (titre VI, art. K sur la justice et les affaires intérieures) [en général et Europol en particulier]. Nous y trouvons les pouvoirs spécifiques des enquêtes proactives qui dépassent le domaine du droit pénal ; plus précisément, l'immigration est identifiée comme un des plus grands risques potentiels contre l'ordre européen à venir, sur le même pied que la grande criminalité.

Schengen, créé en tant que «système précurseur d'Europol ${ }^{26}$, fournit une coopération policière internationale qui comprend différentes méthodes proactives telles que l'observation au-delà des frontières (seulement en cas de présence de soupçon raisonnable) ${ }^{27}$, et des

23 Inzake Opsporing, App. II, 513-515. Les crimes commis par les minorités ethniques sont une question délicate en Hollande. Cet incident a causé des protestations du public, principalement dirigées contre le criminologue en question qui avait été frappé par le grand nombre de Turcs apparemment impliqués dans le trafic de la drogue, et qui fut alors accusé de racisme par les groupes minoritaires après avoir fait part de ses craintes.

Voir, pour une vue historique d'ensemble sur la coopération de la police au niveau européen : Bevers, Joubert, 1994, 19-24.

25 Cf. Bruggeman, 1996; voir aussi Fijnaut, 1993.

26 Bruggeman, 1996.

27 Accords de Schengen, article 40. 
livraisons contrôlées (quand elles sont autorisées par les lois des Etats en question) ${ }^{28}$. L'article 92 du traité introduit néanmoins le SIS (Schengen Information System) : une banque de données informatique partagée, pour faciliter les enquêtes et le contrôle concernant l'ordre et la sécurité publics. La conservation des données se rapporte à des personnes, véhicules et objets en vue d'un contrôle frontalier externe, d'autres contrôles policiers et douaniers, de procédures de visas et de l'application des réglementations sur les étrangers. De plus, l'article 99, paragraphes 2 et 3 , permet la conservation de données sur tout individu dont on peut dire, sur base d'indications concrètes ou d'une impression générale, qu'il est, a été ou pourrait être impliqué dans un crime grave ou, qu'il présente un risque grave ou une menace grave pour la sécurité interne ou externe de l'Etat. L'objet d'un tel stockage de données est de permettre une surveillance non-déguisée ou sous couverture. Tandis que les données sur l'ethnie, les affiliations politiques, la santé, la religion et la sexualité sont exclues, l'immigration et l'émigration sont cependant comprises dans les préoccupations pour l'ordre et la sécurité publics, et le système permet non seulement de surveiller les mouvements des étrangers et des émigrés sur le même pied que des individus suspectés ou reconnus coupables d'infractions, mais il situe carrément les étrangers dans la catégorie des personnes «à risque potentiel grave».

L'article K1 du traité de Maastricht place la politique d'immigration et d'asile sur la liste des 9 zones «d'intérêt commun». Bien que limitant la coopération des forces de l'ordre à la prévention et au combat du terrorisme, au trafic de la drogue et aux formes sérieuses de criminalité internationale, les services «drogues» d'Europol (la première manifestation en vigueur d'Europol, basée sur l'article K 1.9 du traité de Maastricht) ont obtenu une extension de leur mandat qui comprend aussi l'immigration illégale ${ }^{29}$. Quant aux pouvoirs policiers proactifs, le système européen de renseignement ${ }^{30}$ permet la conservation, la modification et l'usage de données sur des personnes présumées en voie de commettre des infractions de la compétence d'Europol. Bien que la limitation aux infractions semble être au moins une sorte de critère, il ne faut pas oublier que les Etats membres sont en passe de criminaliser les émigrants et l'émigration. En effet, dans ce contexte, Swart parle de l'usage de plus en plus fréquent du droit pénal dans le contrôle de l'émigration et du développement d'un plus ou moins cohérent «droit pénal de l'émigration» à partir d'un réseau originellement chaotique de réglementations ${ }^{31}$. En appliquant ce raisonnement dans une perspective internationale, il cite les développements européens qui indiquent une criminalisation systématique de l'émigration ${ }^{32}$. Simultanément, si nous envisageons la tendance naturelle des méthodes policières proactives à dépasser les limites de la procédure pénale, la question devient, une fois encore, plus large. Les événements récents à l'église Saint Bernard de Paris renforcent l'idée que si des populations marginales ou leur famille ont, ou pourraient avoir un statut irrégulier dans un pays particulier, de son côté, le contrôle proactif peut, dans cette situation, viser leur rapatriement et produire ainsi un brouillard confus entre sanctions administratives et pénales.

Si l'on ajoute les occasions de contrôle proactif fournies aux structures administratives de la police dans la nouvelle Europe, il est aisé de voir où mène la dynamique européenne de contrôle ; dans les pays européens, un processus progressif d'exclusion va pousser les

\footnotetext{
$28 \quad$ Accords de Schengen, article 73.

29 Action jointe du ministère de la Justice et du ministère des Affaires Intérieures, le 10 mars 1995.

30 Article 8.1 de la Convention Europol.

31 Swart, 1994.

32 Swart, à paraître, 9-16.
} 
immigrants encore plus loin dans les marges des sociétés où ils se sont établis, créant ainsi un climat de méfiance générale quant à leurs intentions, leur comportement ; autrement dit, ils seront étiquetés comme des cibles légitimes, sujettes à une surveillance continue. Alors que de nombreux règlements, notamment sur le trafic des êtres humains - la prostitution internationale (d'enfants) et l'exploitation d'esclaves au travail -, sont présentées comme faisant partie de «la guerre contre le crime organisé » et donc protégeant aussi les intérêts des immigrants, et que ce type d'abus organisé au niveau international justifie une attention sérieuse, les motifs des gouvernements européens sont plus souvent de maintenir la politique d'immigration que de protéger des individus. Swart remarque que le trafic d'êtres humains est de plus en plus corrélé à l'assistance portée aux immigrants pour entrer clandestinement dans un pays ${ }^{33}$. Tandis que le premier constitue une victimisation, le second est un comportement consensuel. Alors que, en théorie, l'on s'attendrait à ce que les victimes, si possible, informent la police et, au minimum, qu'elle trouve son intervention bienvenue, les individus qui participent à l'entrée clandestine d'immigrants - surtout lorsque leurs raisons ne sont pas économiques (parenté, idéalisme) - ne peuvent être dépistés que grâce à une surveillance et un contrôle permanents.

Un dernier problème à aborder dans le contexte du contrôle proactif international est celui du contrôle et de la surveillance des contrôleurs. La situation en Hollande montre très clairement que les structures administratives existantes (surtout au sein du processus pénal) ne suffisent plus au niveau national. Mais en dehors de l'Etat national, il n'y a aucune structure en place. Bruggeman remarque qu'un puissant dynamisme se développe dans les services anti-drogues européens mais il regrette le manque de composante judiciaire et insiste sur la nécessité de développer des contrôles démocratiques et des normes de conduite légitime, surtout dans le cas des contrôles proactifs ${ }^{34}$. En effet, Europol n'est ni intégré au système de maintien de l'ordre national, ni même n'existe-t-il un quelconque système au niveau européen, et quant aux Comités Exécutifs établis sur la base des accords de mise en application du traité de Schengen, à la Convention de Dublin sur le droit d'asile, et à la Convention sur les frontières externes, ils existent d'une manière similaire dans les limbes institutionnelles et constitutionnelles, en dehors du renvoi à un quelconque contrôle efficace judiciaire ou parlementaire ${ }^{35}$. L'étendue de la protection fournie par la Convention européenne des droits de l'Homme, surtout en ce qui concerne les droits et libertés garantis par les articles 6 et 8, est plus qu'incertaine au regard des transgressions qui peuvent avoir lieu dans le contexte de ce que Curtin nomme des opérations internationales non-identifiées (des organisations indépendantes avec un pouvoir de décision indépendant) qui ne sont pas complices de la CEDH. Ni les accords de mise en application du traité de Schengen, ni la Convention Europol ne font référence à la CEDH et l'avertissement que la justice et les affaires intérieures doivent être traitées en conformité avec la CEDH (article K2.1 du traité de Maastricht) n'a guère de substance puisqu'aucun mécanisme judiciaire externe n'a été créé pour sa mise en application. Curtin entrevoit une solution possible dans le renforcement du rôle de la Cour de Justice pour la protection des droits fondamentaux au sein de l'Union Européenne ${ }^{36}$.

Malgré tout, l'expérience hollandaise suggère que la dynamique des contrôles proactifs non seulement renforce la notion que le secret et l'autonomie par rapport à un contrôle

33 ibidem, 12 ss.

34 Pour une analyse éclairée des problèmes de structures administratives de contrôle et surtout de protection des droits fondamentaux en rapport avec les accords de Schengen et de Maastricht, voir Curtin, 1996. 
hiérarchique et judiciaire, sont les seuls moyens d'obtenir des résultats (une idée qui est en contradiction avec l'accumulation de jurisprudence par la CEDH, qui met l'accent sur la nécessité d'une base légale et sur la faculté pour la défense de contester les preuves), mais aussi encourage un comportement «innovateur» de la part de la police, afin de contourner les contrôles externes. En Belgique, des recherches sur les «zones grises» des activités policières proactives suggèrent l'existence florissante de réseaux officieux internationaux de coopération policière, au sein desquels des organisations privées et des enquêteurs privés jouent un rôle de plus en plus important dans la collecte, l'échange et le blanchiment de renseignements, surtout dans les cas où les réglementations nationales limitent le champ des opérations policières ${ }^{37}$. Il est indéniable que l'unification européenne, avec sa suppression de contrôles frontaliers internes et sa perte des fonctions traditionnelles des frontières, facilitera non seulement le mouvement incontrôlé d'individus à travers le continent mais rendra aussi le contrôle de la criminalité (organisée) internationale et transfrontalière singulièrement plus difficile. Pendant la rédaction de ce texte, en Belgique, une affaire horrible d'abus sexuels et de meurtres d'enfants défraye la chronique, et dans laquelle on trouve de nombreuses suggestions d'un élément organisé au niveau international. Inévitablement, il y a eu des requêtes pour accroître le mandat actuel d'Europol. De tels exemples de l'aspect parfois odieux du crime ne doivent pas nous aveugler quant au fait que le contrôle du crime comporte souvent des pratiques douteuses, ni même nous empêcher de nous demander qui accomplit ces besognes, comment, et jusqu'à quel point nous tenons à ce qu'ils gardent les mains propres.

\author{
Chrisje Brants \\ Pompe Instituut \\ Universiteit Utrecht \\ Boothstraat 6 \\ NL-3512 B.W. Utrecht \\ Stewart Field \\ Cardiff Law School \\ University of Wales \\ PO Box 427 \\ Museum Avenue \\ GB-Cardiff CF1 1XD \\ FieldSA@cf.ac.uk
}

\title{
BIBLIOGRAPHIE
}

BEESTRA T. A. et al., De sociale constructie van georganiseerde misdaad in Nederland, in MOERINGS M., Ed., Hoe punitief is Nederland ?, Arnhem, Gouda Quint, 1994, 237-253.

BEVERS H., JOUBERT Ch., Politiële samenwerking in Europa, Arnhem, Gouda Quint, 1994.

BRANTS C., FIELD S., Participation Rights and Proactive Policing, Convergence and Drift in European Criminal Process, Preadvies No 51, Deventer, Kluwer, 1995.

BRUGGEMAN W., Policing in Europe : A New Wave ?, Conférence à l'Université d'Utrecht, 8 Février 1996.

CURTIN D. M., Criminal Justice, Unidentified International Organisations and Human Rights Protection in Europe, in BRANTS C. H. et al., Eds, Er is meer. Opstellen over mensenrechten in internationaal en nationaal perspectief, Arnhem, Gouda Quint, 1996.

37 Voir De Corte, Van Laethem, Van Outrive, dans ce même numéro. 
DAMAŠKA M. R., The Faces of Justice and State Authority. A Comparative Approach to the Legal Process, New Haven, Yale University Press, 1986.

DELMAS-MARTY M., Ed., The Criminal Process and Human Rights : Toward a European Consciousness, Dordrecht, Martinus Nijhoff, 1995.

FIJNAUT C., The Internationalisation of Police Cooperation in Western Europe, Arnhem, Gouda Quint, 1993.

FIJNAUT C., MARX G. T., Introduction : The Normalization of Undercover Policing in the West. Historical and Comparative Perspectives, in FIJNAUT C., MARX G. T., Eds, Undercover - Police Surveillance in Comparative Perspectives, Den Haag, Kluwer Law International, 1995, 1-16.

GREER S., Supergrasses. A Study in Anti-Terrorist Law Enforcement in Northern Ireland, Oxford, Clarendon Press, 1995.

HOOGENBOOM B., Over de betrekkelijkheid van het debat over opsporingsmethoden. Proactivering in relatie tot bijzondere opsporingsdiensten en particuliere recherche, Delikt en Delinkwent, 1995, 25, 6, 570-593.

Inzake Opsporing. Enquêtecommissie opsporingsmethoden, Bijl. Hand. TK 1995-1996, 24072.

JOUBERT Ch., Undercover Policing - A Comparative Study, European Journal of Criminal Law and Criminal Justice, 1994, $2,1$.

MAGUIRE M., Crime Statistics, Patterns and Trends : Changing Perceptions and their Implications, in MAGUIRE M., MORGAN R., REINER R., Eds, The Oxford Handbook of Criminology, Oxford, Clarendon, 1994, 233-291.

MINISTRY OF JUSTICE, In juiste verhouding. Beleidsvoornemens m.b.t. rechsthandshaving en veiligheid, Den Haag, Juli 1996.

MONTJARDET D., LEVY R., Undercover Policing in France : Elements for Description and Analysis, in FIJNAUT C., MARX G. T., Eds, Undercover - Police Surveillance in Comparative Perspective, Den Haag, Kluwer Law International, 1995, 29-54.

NADELMANN E. A., Cops Across Borders. The Internationalization of U.S. Criminal Law Enforcement, University Park (PA), Pennsylvania State University Press, 1993.

RIEHLE E., Verdacht, Gefahr und Risiko ; der V-mann : ein weiterer Schritt auf dem Wege zu einer anderen Polizei, Kriminologisches Journal, 1985, 17, 1, 44-58.

SWART A. H., Migratiestrafrecht, in BRUINSMA F. et al., Precaire Waarden, Liber Amicorum voor Prof. mr. A.A.G. Peters, Arnhem, Gouda Quint, 1994, 110-134.

SWART A. H., Criminalisering van migratie, à paraître. 